Article

\title{
From Quantum to Cosmological Regime. The Role of Forcing and Exotic 4-Smoothness
}

\author{
Jerzy Król ${ }^{1, \dagger}$, Torsten Asselmeyer-Maluga ${ }^{2, \dagger}$, Krzysztof Bielas ${ }^{1, \dagger}$ and Paweł Klimasara ${ }^{1, *, \dagger}$ \\ 1 Institute of Physics, University of Silesia, Uniwersytecka 4, 40-007 Katowice, Poland; \\ jerzy.krol@us.edu.pl (J.K.); krzysiek.bielas@gmail.com (K.B.) \\ 2 German Aerospace Center, Rutherfordstraße 2, 12489 Berlin, Germany; torsten.asselmeyer-maluga@dlr.de \\ * Correspondence: iriking@wp.pl \\ + These authors contributed equally to this work.
}

Academic Editors: Mariusz P. Dąbrowski, Manuel Krämer and Vincenzo Salzano Received: 30 January 2017; Accepted: 21 March 2017; Published: 27 March 2017

\begin{abstract}
Recently, a cosmological model based on smooth open 4-manifolds admitting non-standard smoothness structures was proposed. The manifolds are exotic versions of $\mathbb{R}^{4}$ and $S^{3} \times \mathbb{R}$. The model has been developed further and proven to be capable of obtaining some realistic cosmological parameters from these exotic smoothings. The important problem of the quantum origins of the exotic smoothness of space-time is addressed here. It is shown that the algebraic structure of the quantum-mechanical lattice of projections enforces exotic smoothness on $\mathbb{R}^{n}$. Since the only possibility for such a structure is exotic $R^{4}$, it is found to be a reasonable explanation of the large-scale four-dimensionality of space-time. This is based on our recent research indicating the role of set-theoretic forcing in quantum mechanics. In particular, it is shown that a distributive lattice of projections implies the standard smooth structure on $\mathbb{R}^{4}$. Two examples of models valid for cosmology are discussed. The important result that the cosmological constant can be identified with the constant curvature of the embedding (exotic $R^{4}$ ) $\rightarrow \mathbb{R}^{4}$ is referred. . The calculations are in good agreement with the observed small value of the dark energy density.
\end{abstract}

Keywords: cosmology and lattice of projections in QM; forcing; exotic 4-smoothness; cosmological constant

\section{Introduction}

It is rather widely accepted that our Universe evolved from an initial quantum state, giving rise to the large scale classical world described by general relativity (GR). The large-scale smoothness of the observed universe is usually taken for granted as some distinguished feature of the classical world. Could the large-scale smoothness be deduced from-or connected with-the initial quantum state? This question is motivated partly by mathematics, where in dimension four there exist a plethora of nonequivalent smoothness structures on open manifolds. Let us mention only the case of the simplest 4-manifold $\mathbb{R}^{4}$ on which one finds continuum many nondiffeomorphic different smoothness structures. On any other $\mathbb{R}^{n}(n \neq 4)$, there exists a unique smoothness structure. Since space-time coordinates are described by the (real smooth functions of) real numbers $\mathbb{R}$, we study the possibility that the real line used in cosmology is an emergent concept and can be described as undergoing several changes during the evolution of the Universe. Thus, we show how $\mathbb{R}$ in the present macroscale form could follow from the formalism of quantum mechanics $(\mathrm{QM})$. The promising mathematical picture formalizing stages of this evolution of parameter space $\mathbb{R}$ is set-theoretic forcing taking place in Boolean-valued models of Zermelo-Fraenkel set theory (ZFC). These particular models are built from quantum-mechanical lattices of projections. An important by-product of this scenario is the change of the smooth geometry of space-time at large scales. Finally, we establish how the above results fit 
with the evolution of the Universe in a cosmological model and help to approach the cosmological constant problem. In particular, a cosmological constant value is calculated in a purely topological way based on deep results in the differential topology of exotic 4-manifolds.

\section{Classical Large-Scale Coordinates from Quantum Regime}

There are many ways in which QM formalism refers to the line of real numbers. One reason is simple: QM describes quantum systems in spacetime. A free particle in the one-dimensional space $\mathbb{R}$ is a good example. A more quantum-mechanical instance would be the continuous spectrum of an operator like the position operator $\hat{Q}$. It is the real line $\mathbb{R}$. The Hilbert space is $L^{2}(\mathbb{R}, \mu)$, where $\mu$ is the Lebesgue measure on $\mathbb{R}$ again. This follows from the fact that the position operator is unbounded and defined on a separable complex Hilbert space $\mathcal{H}$ which has to be infinite dimensional if we want the standard uncertainty relations of position and momentum to be fulfilled. Thus, the only non-empty spectrum of $\hat{Q}$-as the operator of multiplication by $x$ in $L^{2}\left(\mathbb{R}^{1}\right)$-is continuous and equals $\mathbb{R}$. In the case of three-dimensional space $\mathbb{R}^{3}$, the spectrum of the position factorizes into $\mathbb{R} \times \mathbb{R} \times \mathbb{R}$ according to the components of the position operator $\hat{Q}=\left\{\hat{Q}_{i}, i=1,2,3\right\}$. The Hilbert space now reads $L^{2}\left(\mathbb{R}^{3}\right)=L^{2}(\mathbb{R}) \otimes L^{2}(\mathbb{R}) \otimes L^{2}(\mathbb{R})$. All the instances of $\mathbb{R}$ above reflect the obvious fact that we have just "the same" real line $\mathbb{R}$ (up to natural isomorphisms) in every such context. This real line $\mathbb{R}$ is obviously the same (diffeomorphic) as that used in GR for parametrizing the large-scale Universe. In this paper, we demonstrate that some important mathematical facts intervene here. In particular, there exists an interesting relationship between the large and microscale differential structures of spacetime. They can differ non-trivially, and the information about it is encoded in QM formalism.

There is yet another consequence of our analysis. It is the presence of set-theoretic forcing which changes the structure of the real line. Forcing in QM is not quite a new topic. Namely, Wesep [1] and Boos [2] made extended analyses of the local hidden variables program (LHV). It follows that the implementation of LHV requires adding the special "generic filters". Generic filters are precisely those which are known in formal set theory and the technique of forcing. Whenever a nontrivial generic filter $G$ is added to a model ZFC $M$, it leads to another model of ZFC, $M[G]$, extended by forcing. However, such forcing extensions change the real line $R$ in $M$ into the extended real line $R[G]$ in $M[G]$. This change of the real line is what matters to us here. By Kochen-Specker theorem LHV cannot be realized in QM for Hilbert spaces of dimensions greater than two [3]. We do not meet these constraints here, since our results do not rely in any sense on the realizability of the LHV program. Moreover, forcing can be regarded as a technique for exploring the structure of the real line rather than proving independence results in formal set theory. Set-theoretic forcing as a tool in QM has already been identified and used by several authors, such as P. Benioff [4], G. Takeuti [5], M. Ozawa [6,7], W. Boos [2], R. Van Wesep [1], and the present authors [8-12].

In the next section we will show that QM formalism on Hilbert spaces refers to models of ZFC and that the models support non-trivial forcing. In Section 4, we identify the forcing as random forcing defined on atomless measure algebras $\operatorname{Bor}\left(\mathbb{R}^{n}\right) / \mathcal{N}$ of Borel sets in $\mathbb{R}^{n}$ modulo measure zero sets. This forcing is always present when passing from micro QM realm into the large-scale structure of the Universe. The real line is changed by the forcing such that we have the mechanism for canceling the vacuum energy contributions from the zero-modes of quantum fields. In Section 5 we show that the models of ZFC localized at the lattice of projections determine the necessary exotic smoothness on $\mathbb{R}^{n}$ at macroscales. It is possible only for $n=4$, which is the reason for the dimensionality of the large-scale spacetime emerging from the QM regime. Section 6 presents an example of a Schwarzschild black hole which is described on exotic Kruskal manifold $S^{2} \times \mathbb{R}^{2}$ [13]. In Section 7, we give a rough presentation of our recent findings on topological calculations of some cosmological parameters like the value of cosmological constant or rate of inflation in the model where smoothness of spacetime is exotic. The calculations are rooted in the special role of hyperbolic three- and four-dimensional geometry involved in the very structure of exotic smooth 4-manifolds. 


\section{The Lattice of Projections $\mathbb{L}$}

Let $\mathcal{H}$ be a separable Hilbert space. The set of all projections on closed linear subspaces of $\mathcal{H}$ gives rise to an atomic complete orthomodular lattice $(\mathbb{L}(\mathcal{H}), \wedge, \vee) \equiv \mathbb{L}$, where $P_{1} \wedge P_{2}$ is defined to be $M_{1} \cap M_{2}$ and $P_{1} \cup P_{2}$ 一the least closed subspace containing $M_{1} \cup M_{2}$. Here $M_{1}, M_{2}$ are subspaces corresponding to $P_{1}, P_{2}$ [5]. Since $\mathbb{L}$ is not distributive if $\operatorname{dim} \mathcal{H}>1$, it has been recognized that $\mathbb{L}$ cannot serve as a source of classical propositional logic [14].

One of the possible strategies is to proceed with the set $\mathrm{BSub}(\mathbb{L})$ of Boolean subalgebras $\mathcal{B} \subseteq \mathbb{L}$, partially ordered by set inclusion. Since $B S u b(\mathbb{L})$ can be provided a meet-semilattice structure [15], every chain is well-ordered in particular, and by Zorn's lemma, every $B \in \operatorname{BSub}(\mathbb{L})$ is contained in a maximal $B^{\prime} \in \operatorname{BSub}(\mathbb{L})$. Moreover, every maximal $B^{\prime} \in \mathrm{BSub}(\mathbb{L})$ is complete, since it is subcomplete [16]. Such complete maximal Boolean subalgebras (later also called blocks) of $\mathbb{L}$ play an essential role in constructing Boolean-valued models upon $\mathbb{L}$ [5]. The versatility of the above approach relies on the general properties of self-adjoint operators in the following sense.

Lemma 1. ([5] Lemma 1.1 p. 8) For every family $\left\{A_{\alpha} \mid \alpha \in I\right\}$ of self-adjoint pairwise commuting operators, there exists a complete Boolean algebra of projections $B$ such that given the spectral decompositions of each $A_{\alpha}$

$$
A_{\alpha}=\int \lambda \mathrm{d} E_{\lambda}^{\alpha},
$$

it holds that $\forall \alpha \in I\left(\mathrm{~d} E_{\lambda}^{\alpha} \in B\right)$.

In what follows, a self-adjoint operator is said to be in a Boolean algebra $B$ when its spectral decomposition is a subset of $B$. Then, as a consequence of Lemma 1 and Zorn's lemma, every family $\left\{A_{\alpha} \mid \alpha \in I\right\}$ of self-adjoint pairwise commutable operators is contained in at least one block $B \subseteq \mathbb{L}$.

Let us provide some examples for the above remarks. ${ }^{1}$

Proposition 1. Let $\mathcal{H}=L^{2}(\mathbb{R}, \mathrm{d} x)$, and let $B_{Q} \subseteq \mathbb{L}$ be the block containing a position operator $Q$ on $\mathcal{H}$. Then, $B_{Q}$ is atomless.

Proof. It can be shown that $Q$ is multiplicity-free, hence it defines a maximally commuting system of observables on $L^{2}(\mathbb{R}, \mathrm{d} x)$ [17]. Thus, every self-adjoint operator $A$ that commutes with $Q$ is a measurable function of $Q$ (i.e., $A=f(Q)$ ). As a consequence of the spectral theorem, the spectral decomposition of $f(Q)$ coincides with $\left\{Q_{\lambda}\right\}$. However, $\left\{Q_{\lambda}\right\}=\left\{\chi_{\Delta}\right\}$ with measurable $\Delta \subseteq \mathbb{R}$, and $\left\{\chi_{\Delta}\right\}$ is isomorphic to the measure algebra on $\mathbb{R}$; i.e., $\operatorname{Bor}(\mathbb{R}) / \mathcal{N}[5]$, which proves $\left\{Q_{\lambda}\right\}$ to be an atomless block.

Equivalently, the same holds for the block containing a momentum operator $P$ on $L^{2}(\mathbb{R}, \mathrm{d} x)$. The following fact shows that given a self-adjoint operator $A$, the atomicity of a block containing $A$ is not an invariant of $A$ in general.

Proposition 2. Let $\mathcal{H}=L^{2}\left(\mathbb{R}^{3}, \mathrm{~d}^{3} x\right)$ and let $B_{H} \subseteq \mathbb{L}$ be a block containing a free-particle Hamiltonian $H=P_{1}^{2}+P_{2}^{2}+P_{3}^{2}$. Then, $B$ is either atomless or contains an atom.

Proof. Since $H$ commutes with components $P_{i}$ of momentum operator and $\left\{P_{i} \mid i=1,2,3\right\}$ define a maximally commuting system of observables on $\mathcal{H}=L^{2}\left(\mathbb{R}^{3}, \mathrm{~d}^{3} x\right), H$ is contained in an atomless block. On the other hand, as a consequence of [18], (Theorem II.4)—provided $H$ does not set the maximally commuting system-there is a self-adjoint operator $A$ with pure point spectrum such that $\{H, A\}$ is

1 We would like to thank Valter Moretti for helpful discussion on this subject. 
already commuting and maximal. In this case, $H$ is contained in a block with atoms corresponding to one-dimensional spectral projections of $A$.

\section{The Physics of Forcing}

The equivalence of the method of forcing in formal set theory as a method of proving independence results with its formulation in Boolean-valued models $V^{B}$ ( $B$ is a complete Boolean algebra) was established by Scott and Solovay soon after the invention of forcing [19] (p. 18). The following fact gives the necessary condition for forcing with a Boolean algebra $B$ to be nontrivial (i.e., to extend the ground ZFC universe $V$ properly), and explains the importance of non-atomicity of $B$.

Lemma 2. ([20] Proposition 2.1) Suppose there exists a generic ultrafilter $U$ on $B$ over $V$. Then, $U \notin V$ if and only if $B$ is atomless.

Let $\mathcal{H}$ be a separable Hilbert space on which position $Q$ and momentum $P$ operators are represented in the usual way. Let $B_{Q}, B_{P} \subseteq \mathbb{L}$-blocks containing $Q$ and $P$, respectively. Obviously, $P \notin B_{Q}$ and $Q \notin B_{P}$. The real numbers $R_{Q}, R_{P}$ in Boolean-valued models of ZFC Sh $\left(B_{Q}\right), \operatorname{Sh}\left(B_{P}\right)$ are in $1-1$ correspondence with self-adjoint operators in $B_{Q}, B_{P}$, respectively. This is the important result of Takeuti [5]. We call $R_{Q}, R_{P}$ the Boolean quantum real numbers.

Proposition 1 states that the algebras $B_{Q}, B_{P}$ are atomless, and hence by Lemma 2 they support a nontrivial forcing. Suppose there exist generic ultrafilters $U l_{Q}, U l_{P}$ on $B_{Q}, B_{P}$ over universes $V_{Q}, V_{P}$, respectively. The following factorizations lead to two-valued models of ZFC (e.g., [19,20]):

$$
\operatorname{Sh}\left(B_{Q}\right) / U l_{Q}=V_{Q}\left[U l_{Q}\right], \operatorname{Sh}\left(B_{P}\right) / U l_{P}=V_{P}\left[U l_{P}\right],
$$

and $U l_{Q} \notin V_{Q}, U l_{P} \notin V_{Q}$. Then, $R\left[U l_{Q}\right], R\left[U l_{P}\right]$ are objects of real numbers in the above two-valued models (quantum real numbers).

Proposition 2 shows that free-particle energy $H$ possibly lives in a block $B_{H} \subset \mathbb{L}$ that contains at least one atom. From Lemma 2 it follows that forcing in the case of Boolean-valued model $\operatorname{Sh}\left(B_{H}\right)$ is trivial; i.e.,

$$
\operatorname{Sh}\left(B_{H}\right) / U l_{H}=V_{H}\left[U_{H}\right]=V_{H}, R\left[U l_{H}\right]=R_{H}
$$

for every generic ultrafilter in $B_{H}$ over $V_{H}$. This simply means that $U l_{H} \in V_{H}$ and forcing has to be trivial, even if $U l_{H}$ would be generic over $\operatorname{Sh}\left(B_{H}\right)$.

Let us suppose that (large-scale) gravitational fields propagate in the spacetime which is parametrized by real numbers in a model extended by a nontrivial forcing. The case of the energy operator discussed above shows that some forms of energy - namely those whose self-adjoint operators give rise to the non-atomless Boolean algebras as in Proposition 2-propagate in spacetime parametrized by the reals not extended by forcing. This cosmological model was indeed recently studied [8-10], where it was proposed that zero-energy modes of energy of quantum fields propagate in spacetime which is parametrized in a ZFC model not extended by forcing. Here, however, $B_{Q}, B_{P}$ lead to the nontrivial extension of the model and the real line. We propose GR to describe gravity living and propagating in such models. This discrepancy has important physical consequences.

First we recognize general forcing appearing above as random forcing; i.e., the Boolean algebras in question are in fact measure algebras $\operatorname{Bor}\left(\mathbb{R}^{n}\right) / \mathcal{N}$, where $\operatorname{Bor}\left(\mathbb{R}^{n}\right)$ are Borel subsets of $\mathbb{R}^{n}$ and $\mathcal{N}$ is an ideal of measure zero sets. It holds that

Theorem 1. $B_{Q} \simeq \operatorname{Bor}\left(\mathbb{R}^{n}\right) / \mathcal{N}$ for some $n \in \mathbb{N}$.

Proof. The isomorphism of $B_{Q}$ and $\operatorname{Bor}(X) / \mathcal{N}$ was established in [5], where $(X, \mu)$ is some measure space. This is the direct consequence of the spectral theorem [5] (Th. 6.1, p. 68), where the Hilbert space representing a family of commuting self-adjoint operators as multiplication by measurable function 
operators is $L^{2}(X, \mu)$. This Hilbert space is separable and infinite dimensional, and so it is isometric isomorphic to a $L^{2}\left(\mathbb{R}^{n}, \mathrm{~d}^{n} x\right)$ for some $n \in \mathbb{N}$, as follows from the Riesz theorem. From Proposition 1 we know that $B_{Q}$ is atomless in $L^{2}\left(\mathbb{R}^{n}, \mathrm{~d}^{n} x\right)$. The isomorphism of Hilbert spaces preserves the maximal Boolean algebras of projections.

Next we quote some known properties of random forcing (e.g., [21]).

Lemma 3. $B_{n}=\operatorname{Bor}\left(\mathbb{R}^{n}\right) / \mathcal{N}$ is atomless for every $n \in \mathbb{N}$. Every nontrivial forcing extension of the real line $R$ in $V$ to $R[U l]$ in $V[U l]$, for $U l \in B_{n}$ generic over $\operatorname{Sh}\left(B_{n}\right) \simeq V^{B_{n}}$ is such that all measurable subsets of $R$ in the extended model $V[U l]$ have measure zero.

Recall that zero-energy modes of quantum fields propagate in the ZFC model $V$, while both higher modes of quantum fields and large-scale gravity propagate in the extended model $V[U l]$. This is the reflection of the fact that zero-energy modes have pure quantum-mechanical origin (i.e., they vanish classically), while higher modes give rather classical contributions (e.g., [22] (p. 28)). Namely, the vacuum energy density has two components in quantum field theory: bare density without any quantum corrections and quantum zero-energy modes fluctuations of all quantum fields. The total zero-energy modes contribution of any quantum field reads $\sum_{i} \frac{1}{2} \omega_{i}$. In the continuum limit, one gets the energy density of zero-energy modes of a free field as $\int_{\mathbb{R}^{3}} \frac{\mathrm{d}^{3} k}{(2 \pi)^{3}} \frac{\sqrt{\mathbf{k}^{2}+m^{2}}}{2}$. Without any cutoff imposed, this integral is UV-divergent. However, in our model, it holds that $[8,9]$

Theorem 2. Zero-energy modes of quantum fields have vanishing contributions to the gravitational vacuum energy.

Proof. From Lemma 3, we see that every integral $\int_{R^{3}}(\cdot) \mathrm{d} x[U l]^{3}$ calculated in the extended model $V[U l]$ vanishes $(V \ni R \subset R[U l] \in V[U l])$. The reason is that every measurable subset of $R^{3}$ has measure 0 in $R[U l]^{3}$, and $R$ is a meagre subset of $R[U l]$. In particular, given a particle of mass $m$, its zero-energy modes contributions to the energy density are given by the vanishing integral $\int_{R^{3} \subset R[U l]^{3}} \frac{\mathrm{d}^{3} k}{(2 \pi)^{3}} \frac{\sqrt{\mathbf{k}^{2}+m^{2}}}{2}[8,9]$.

The above result means that in our forcing-based model the cosmological constant derived from zero-energy modes of quantum fields has to vanish, contrary both to quantum field-theoretic calculations [23] and to experimental facts [24]. However, in what follows we will see that the relation of quantum-mechanical lattice $\mathbb{L}$ and large-scale world gives rise to a surprising solution.

\section{Macroscopic Smoothness from $\mathbb{L}$}

Let $\mathbb{R}$ be a set of all real numbers. It is a Dedekind complete ordered field, and as such all its (Archimedean) models are isomorphic. This explains an unambiguity of the symbol $\mathbb{R}$ (up to the isomorphisms). The Dedekind completeness is a second-order property-unlike all the axioms of ZFC, which are first-order. So far, we have considered a change of models of ZFC due to the random forcing. In this section, we want to analyse yet another modification of real numbers. The real numbers inside first-order models of ZFC are now replaced by $\mathbb{R}$ - the Dedekind complete, ordered, second-order field. This operation is to be derived from the lattice of projections $\mathbb{L}$. The following definitions clarify the terminology.

\section{Definition 1.}

(a) The substitutions $R\left[U l_{Q}\right] \rightarrow \mathbb{R}$ and $R\left[U l_{P}\right] \rightarrow \mathbb{R}$ are called large-scale classical limits of (quantum) real numbers $R\left[U l_{Q}\right]$ and $R\left[U_{P}\right]$, respectively. We write $\mathbb{R} / R\left[U l_{Q}\right]$ and $\mathbb{R} / R\left[U l_{P}\right]$ for the substitutions.

(b) Similarly, the substitution $R\left[U l_{A}\right] \rightarrow \mathbb{R}$ is a large-scale classical limit of the real numbers $R\left[U l_{A}\right]$ from the model $\mathrm{Sh}\left(B_{A}\right) / U l_{A}$ for a self-adjoint operator $A$; i.e., $\mathbb{R} / R\left[U l_{A}\right]$. 
Note that given any self-adjoint operator $A$ on a (separable) Hilbert space, its maximal complete Boolean algebra of projections $B_{A}$ generally need not be atomless. In this case, a generic ultrafilter which is not in the ground model need not exist. Then, taking any ultrafilter $U l_{A}$ on $B_{A}$ over $V$, the factorization reads $\operatorname{Sh}\left(B_{A}\right) / U l_{A} \simeq V\left[U l_{A}\right] \simeq V$, since $U l_{A} \in V$. This is the direct consequence of Lemma 2. Consequently, $R\left[U l_{A}\right] \simeq R$. Again, the substitution $R \rightarrow \mathbb{R}$ is the classical limit of $R$.

Let $I_{\mathbb{L}}$ be the set where each $\alpha \in I_{\mathbb{L}}$ is some complete Boolean algebra $B_{\alpha}$ of projections from $\mathbb{L}$. Let $\mathbf{b a}(\mathbb{L})$ be a set of all such maximal complete Boolean algebras. It holds that $\mathbf{b a}(\mathbb{L}) \subset I_{\mathbb{L}}$.

Let $U_{\alpha} \stackrel{\text { diff. }}{\rightarrow} \mathbb{R}^{n}, \alpha \in \mathbf{b a}(\mathbb{L})$ be a local regular open cover of an $n$-dimensional topological manifold $M_{\mathbb{L}}^{n}$, and each $U_{\alpha}$ is the classical large-scale limit of some $R_{\alpha}^{n}$. We require the cover $\mathcal{U}=\left\{U_{\alpha} \mid \alpha \in \mathbf{b a}(\mathbb{L})\right\}$ of $M_{\mathbb{L}}^{n}$ to have the following properties:

$$
\begin{gathered}
\left.\exists \mathcal{U} \forall U_{\alpha} \in \mathcal{U} \exists \alpha \in \mathbf{b a}(\mathbb{L})\right\}\left(\mathcal{U}_{\alpha} \stackrel{\text { diff. }}{\rightarrow} \mathbb{R}^{n} / R_{\alpha}^{n}\right), \\
\forall \mathcal{K} \text { an open cover of } M_{\mathbb{L}}^{n}(\mathcal{U} \subset \mathcal{K} \Rightarrow \bigcup \mathcal{U} \notin \mathcal{K}), \\
\exists \alpha, \beta \in \mathbf{b a}(\mathbb{L})\left(U_{\alpha}, U_{\beta} \in \mathcal{U} \Rightarrow U_{\alpha} \cup U_{\beta} \notin \mathcal{U}\right) .
\end{gathered}
$$

Recall that the lattice $\mathbb{L} \equiv \mathbb{L}(\mathcal{H})$ has to be defined over infinite dimensional Hilbert space $\mathcal{H}$ in order to represent the position-momentum uncertainty relations. Hence, $\mathbb{L}$ is not distributive and cannot be reduced to any single Boolean algebra of projections. For that reason, one can take $\alpha=B_{Q}$ and $\beta=B_{P}$ in (3).

Definition 2. If $\left\{U_{\alpha}, \alpha \in \mathbf{b a}(\mathbb{L})\right\}$ as above is a smooth regular open cover of a smooth n-dimensional manifold $M_{\mathbb{L}}^{n}$, then the pair $\left(M_{\mathbb{L}}^{n},\left\{U_{\alpha}, \alpha \in \mathbf{b a}(\mathbb{L})\right\}\right)$ is called a smooth manifold large-scale classical limit (or classical limit for short) of the lattice $\mathbb{L}$.

It is obvious that many different smooth manifolds $M^{n}$ (in various dimensions) generally exist as candidates for large-scale classical limits of $\mathbb{L}$. However, one case is distinguished-namely, $M^{n}=\mathbb{R}^{n}$. It also distinguishes the physical dimension four.

Theorem 3. If $\mathbb{R}^{n}$ is a (smooth manifold large-scale) classical limit of the QM lattice $\mathbb{L}$, then it has to be an exotic $\mathbb{R}^{4}$.

Proof. Let $\mathcal{K}$ be a regular topological open cover of the smooth $\mathbb{R}^{n}$ large enough to contain the subcover $\mathcal{U} \subset \mathcal{K}$. We will show that $\mathcal{U}$, being a smooth cover of $\mathbb{R}^{n}$ and fulfilling Equations (1)-(3) has to be exotic smooth. Each $U_{\alpha} \in \mathcal{U}$ is diffeomorphic to $\mathbb{R}^{n}$, which resulted as a substitution $\mathbb{R}^{n} / R_{\alpha}^{n}$ (Equation (1)). Here $R_{\alpha}^{n}$ is the $n$-product of the real line in the two-valued model Sh $(\alpha) / U l$ for some ultrafilter in $\alpha, \alpha$ is a maximal complete Boolean algebra of projections in $\mathbb{L}$. Properties (2) and (3) require that for some subfamily $\mathcal{A}=\left\{\alpha_{i} \mid i \in J \subset \mathbf{b a}(\mathbb{L})\right\} \subset \mathcal{U}$, it holds $\cup \mathcal{A} \notin \mathcal{U}$. It follows that indeed $\cup \mathcal{U} \notin \mathcal{U}$. However, $\cup \mathcal{U} \stackrel{\text { top }}{\simeq} \mathbb{R}^{n}$, since $\mathcal{U}$ is a topological cover of $\mathbb{R}^{n}$. It follows that $\bigcup \mathcal{U} \not \mathbb{R}^{n}$ as smooth manifolds. However, $\cup \mathcal{U}$ is a smooth manifold which is topologically $\mathbb{R}^{n}$ and nondiffeomorphic to $\mathbb{R}^{n}$. Thus, $\cup \mathcal{U}$ is exotic smooth $\mathbb{R}^{n}$. The only possibility is exotic $\mathbb{R}^{4}$.

Note that the conditions (2) and (3) are fulfilled automatically for any compact $M^{n}$, since $\cup \mathcal{U} \simeq M^{n} \notin \mathcal{U}$ and every $\mathcal{U}_{\alpha} \in \mathcal{U}$ is diffeomorphic to $\mathbb{R}^{n}$.

Corollary 1. The $Q M$ lattice of projections $\mathbb{L}$ is the source of a non-vanishing large-scale curvature on $\mathbb{R}^{4}$. This curvature cannot be removed by any coordinate diffeomorphism of $\mathbb{R}^{4}$.

Proof. Every exotic $\mathbb{R}^{4}$ has to be curved Riemannian smooth manifold topologically equivalent to $\mathbb{R}^{4}$, because if $\mathbb{R}^{4}$ were flat, it would be diffeomorphic to a one-patch $\mathbb{R}^{4}$, which is the standard smooth $\mathbb{R}^{4}$. 
Since any exotic $\mathbb{R}^{4}$ is not diffeomorphic to the standard 4-space, there does not exist any coordinate diffeomorphism resulting in the standard flat $\mathbb{R}^{4}$.

We call the lattice $\mathbb{L}$ of projections Boolean whenever it is reduced to a single maximal complete Boolean algebra. Obviously, a Boolean lattice is distributive and cannot represent the position-momentum uncertainty relations.

Theorem 4. Let the lattice $\mathbb{L}$ be Boolean. Then, the smoothness structure of its manifold classical large-scale limit $\mathbb{R}^{n}$ is the standard one (including the $n=4$ case).

Proof. ba $(\mathbb{L})$ is a one-element set; i.e., $\{\alpha\}=\mathbf{b a}(\mathbb{L})$. Since $U_{\alpha} \stackrel{\text { diff. }}{\simeq} \mathbb{R}^{n}$ is also the only element of $\mathcal{U}$, it follows that the conditions (2) and (3) are not fulfilled and $\cup \mathcal{U} \simeq \mathcal{U}_{\alpha} \in \mathcal{U}$. Hence, the standard smooth $\mathbb{R}^{n}$ is a possible choice.

We see that the nondistributivity of the lattice $\mathbb{L}$ and its non-boolean quantum structure is crucial for the large-scale exotic 4-smoothness. It also indicates the dimension four as the dimension of a smooth large-scale world.

In the following sections we will demonstrate how the modification of differential large scale structure of spacetime by QM works in two physical models. The models deal with cosmological singularities, both having quantum-gravitational character; therefore, $\mathrm{QM}$ is involved here. One is the singularity of a Schwarzschild black hole, the other-the initial singularity in the simplified Friedmann-Lemaître-Robertson-Walker (FLRW) cosmology.

\section{Schwarzschild Solution and Exotic $S^{2} \times \mathbb{R}^{2}$}

Let us consider the Schwarzschild metric around a black hole in the Kruskal presentation

$$
d s^{2}=\frac{2 M^{3} e^{-r / 2 M}}{r}\left(-d u^{2}+d v^{2}\right)+r^{2} d \Omega^{2},
$$

where $d \Omega^{2}=d \theta^{2}+\sin ^{2} \theta d \phi^{2}$ is the standard spherical metric on $S^{2}$, and $\left(\frac{r}{2 M}-1\right)\left(e^{r / 2 M}\right)=v^{2}-u^{2}$ are coordinates on $\mathbb{R}^{2}$. Thus, the Kruskal coordinates describe the manifold $S^{2} \times \mathbb{R}^{2}=\{(u, v,(\theta, \phi))$ : $\left.u^{2}-v^{2}<1,(\theta, \phi) \in S^{2}\right\}$.

From the point of view of GR, the singularity of the Schwarzschild black hole is Weyl-type, since the Weyl tensor diverges [25] (p. 146). The consistent complete description of the singularity requires a theory of quantum gravity (QG). Certainly, we do not yet have such full such quantum gravitational description; still, it is natural to assume that the singularity cannot be completely described only within GR. The form of energy and matter due to this QG regime follows the QM rules. Our analysis of the previous sections thus applies. The QM formalism on a Hilbert space modifies the entire large-scale smoothness structure of the spacetime in which the Schwarzschild solution is considered.

First, note that $S^{2} \times \mathbb{R}^{2}$ is a submanifold of $\mathbb{R}^{4}$; i.e.,

$$
S^{2} \times \mathbb{R}^{2}=\mathbb{R}^{4} \backslash D^{3} \times \mathbb{R},
$$

where $D^{3}$ is a 3-disk. Thus, we have a formal representation of the Schwarzschild singularity as placed in the complement of $D^{3} \times \mathbb{R}$ in $\mathbb{R}^{4}$.

Second, let certain energy/matter from the singular region have usual QM description on a Hilbert space of states. It is a minimal quantum requirement for such singularity present in classical spacetime whose description, however, transcends it. So, let $\mathcal{H}$ be a Hilbert space of states for such energy-matter with $\mathbb{L}$ the lattice of projections. Again, $\mathbf{b a}(\mathbb{L})$ is the set of all complete Boolean algebras of projections from $\mathbb{L}$. The smooth cover of $\mathbb{R}^{4}$ which is determined by $\mathbb{L}$ fulfills the conditions (1)-(3). Then, Theorem 3 gives the modification of the large-scale smoothness structure of $\mathbb{R}^{4}$, which agrees with the presence of such quantum sources. 
Note that in GR, energy-matter sources modify the stress energy tensor $T_{\mu v}$, and the corresponding curvature modification of spacetime follows. The presence of QM sources as above also modifies the smoothness of spacetime at large scales in such a way that it cannot be diffeomorphic to the initial smoothness.

Given the Kruskal coordinates manifold $S^{2} \times \mathbb{R}^{2} \subset \mathbb{R}^{4}$, the modified exotic smoothness of $\mathbb{R}^{4}$ enforces the exotic smoothness on $S^{2} \times \mathbb{R}^{2}$ according to the results in the previous section. It is a mathematical fact that such exotic $S^{2} \times \mathbb{R}^{2 \prime}$ s exist. These exotic $S^{2} \times \mathbb{R}^{2 \prime}$ s were analysed in $[13,26,27]$, and are called exotic Schwarzschild black holes. The peculiar feature of black holes with exotic Kruskal manifold is their non-global product $S^{2} \times \mathbb{R}^{2}$. This means that backward prolongation of smooth Kruskal coordinates meets obstructions which usually are interpreted as, for example, additional external sources [13]. This change of smoothness structure in $4 \mathrm{D}$ is the result of taking together the spacetime description with unavoidable QM one.

\section{FLRW Cosmology with Initial Singularity}

Let us consider the cosmological model of the evolution of the Universe based on the spacetime $S^{3} \times \mathbb{R}$. It is a classical model of the FLRW closed universe where the initial singularity is situated in the region (point) where $S^{3}$ shrinks to zero size. In fact, any FLRW model with ordinary matter with pressure $p$ and density $\rho$ gives rise to the Ricci singularity [25] (p. 146). Certainly, a full and consistent physical and mathematical description of the singularity must refer to QM and QG. Even outside of the singularity, still in the Planck epoch, one cannot neglect quantum description. Large-scale homogenous and smooth spacetime evolves entirely from the singularity. So, whenever we apply the standard formalism of QM based on the Hilbert space and the lattice $\mathbb{L}$, then, according to Section 5, the large-scale smooth structure is modified. The resulting smoothness in this case is exotic $S^{3} \times \mathbb{R}$. For our purposes it can be seen as induced by exotic $\mathbb{R}^{4}$ so the analysis of Section 5 is valid. To see this, note that $S^{3} \times \mathbb{R}$ is a smooth submanifold of $\mathbb{R}^{4}$. Simply it holds $S^{3} \times \mathbb{R} \simeq \mathbb{R}^{4} \backslash\{p t$. $\}$. In the case of exotic $S^{3} \times \mathbb{R}$, it can still be considered as smoothly embedded in exotic $\mathbb{R}^{4}$. This embedded exotic $S^{3} \times \mathbb{R}$ is the exotic topological end of some exotic $\mathbb{R}^{4}[28]$.

One important feature of an exotic open 4-manifold is the radical modification of the smooth time evolution. The cross-sections $S^{3} \times\left\{t_{i}\right\}, \forall t_{i} \in \mathbb{R}$ are replaced by wildly embedded $S^{3}$ at $t_{0}$ evolving smoothly into the cross-section $\Sigma_{t_{1}} \subset S^{3} \times \Sigma \mathbb{R}$; i.e., a smooth 3-manifold non-homeomorphic to $S^{3}$. Here $S^{3} \times_{\Sigma} \mathbb{R}$ denotes the exotic $S^{3} \times \mathbb{R}$ with the cross-section indicated. As shown by Freedman, this is the defining property of exotic $S^{3} \times \mathbb{R}$ where $\Sigma$ is some homology 3-sphere [28]. These modifications allow for direct calculation of certain cosmological effects of exotic smoothness structures on $\mathbb{R}^{4}$ via exotic smoothness of $S^{3} \times \mathbb{R} \subset \mathbb{R}^{4}$ [29].

In [29], we considered one exotic $S^{3} \times \mathbb{R}$ with two topology changes of the 3-manifold $S^{3} \times_{\Sigma} \mathbb{R}$ : wild $S^{3} \stackrel{1}{\rightarrow} \Sigma \stackrel{2}{\rightarrow} P \# P$. Here $P$ is the Poincare 3-homology sphere and $P \# P$ the direct sum of two copies of them. This topology change is a modification of the three-dimensional spatial spaces, while the 4-manifold has constant topology with an exotic smoothness structure. In this model, one is able to calculate cosmological parameters which are usually not determined in the standard $\Lambda$ CDM (Lambda cold dark matter) model [29].

For that purpose, we use the defining property of a small exotic $R^{4}$. These exotic $R^{4}$ s are all embeddable as open subsets in the standard $\mathbb{R}^{4}$. As we already noted in Section 5 , the Riemann tensors

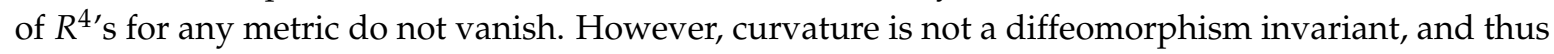
varies for different embeddings $R^{4} \hookrightarrow \mathbb{R}^{4}$. In particular, every embedding realizes a relatively constant negative scalar curvature of some $R^{4}$ embedded in $\mathbb{R}^{4}$. The constancy of the curvature is also known as Mostow rigidity $[29,30]$. Then, we have one Friedmann equation $(\dot{a} / a)^{2}=$ const $>0$, which defines a cosmological constant (CC). 
The 3-homology sphere $\Sigma$ can be chosen as the Brieskorn sphere $\Sigma(2,5,7)$. It is a three-dimensional submanifold of $\mathbb{C}^{3}$ defined as follows:

$$
\Sigma(2,5,7)=\left\{\left.(x, y, z) \in \mathbb{C}^{3}\left|x^{2}+y^{5}+z^{7}=0,\right| x\right|^{2}+|y|^{2}+|z|^{2}=1\right\} .
$$

Then, as shown in [29], topology change $S^{3} \rightarrow \Sigma(2,5,7)$ is described by the scalar field $\phi$ given by the Lagrangian

$$
\mathcal{L}=R+\partial^{\mu} \phi \partial_{\mu} \phi+\frac{1}{8 \alpha}(1-\exp (-\phi))^{2},
$$

which is transformed to the usual Starobinsky Lagrangian [31]

$$
\mathcal{L}=R+\alpha \cdot R^{2} .
$$

Here $R$ is the scalar curvature of (part of) $S=S^{3} \times_{\Sigma(2,5,7)} \mathbb{R}$. Now, performing some smooth 1,2-handles cancellation in the exotic $S$, due to the change $S^{3} \rightarrow \Sigma(2,5,7)$, one gets an expansion

$$
a=a_{0} \cdot \exp \left(\frac{\theta}{2}\right)
$$

where $\theta$ is a parameter which will be determined as a topological invariant, $a_{0}$ is the diameter of $S^{3}$, and $a$ is the characteristic length of the hyperbolic sphere $\Sigma(2,5,7)$. Considering the second topology change $\Sigma(2,5,7) \rightarrow P \# P$ and $a_{0} \sim L_{P}$ 一the Planck length, one calculates the expansion purely topologically:

$$
a=L_{P} \cdot \exp \left(\frac{3}{2 \cdot C S(\Sigma(2,5,7))}+\frac{3}{2 \cdot C S(P \# P)}\right) \simeq L_{P} \cdot 2,2 \cdot 10^{59} \simeq 10^{9} \mathrm{ly} .
$$

Here CS $(\cdot)$ refers to the Chern-Simons invariant of the hyperbolic 3-manifolds, which in our case read

$$
C S(\Sigma(2,5,7))=\frac{9}{280}, C S(P \# P)=\frac{1}{60} .
$$

This is a realistic expansion scale for the inflation which agrees with the Planck mission. The topological calculation of the value of the cosmological constant as the constant curvature of the embedding $R^{4} \hookrightarrow \mathbb{R}^{4}$ follows

$$
C C=\frac{1}{a^{2}}=L_{P}^{-2} \exp \left(-\frac{3}{C S(\Sigma(2,5,7))}-\frac{3}{C S(P \# P)}\right) .
$$

This value expressed as a fraction of the critical density (taking the recent value of the Hubble constant $H_{0}$ from the Planck mission [24]) reads:

$$
\Omega_{\Lambda}(C C)=0.6836,
$$

which is significantly close to the Planck result $\Omega_{\Lambda}=0.6911 \pm 0.0062$.

\section{Discussion}

We have presented a cosmological model which coherently allows the derivation of some cosmological parameters. The model is based on the natural supposition that the evolution of the Universe begins with the quantum regime described by the Hilbert space formalism of QM. The lattice of projections $\mathbb{L}$ serves as the initial data of the model. We showed that for infinite dimensional Hilbert space $\mathcal{H}$, the lattice $\mathbb{L}$ determines the line of real numbers and set-theoretic forcing which modifies this line. The forcing is recognized as the random forcing. These data are usually omitted in cosmological model-buildings. However, as we showed, the random forcing changes the structure of the real line 
when passing from the micro to the macroscales of the Universe. The extension of the line results in the vanishing of the contributions of the zero-points energies of quantum fields to the vacuum energy.

Another change of the structure of the real line occurs during the switch of descriptions from the first-order ZFC theory to the second-order theory of complete ordered fields. This results in the appearance of the large-scale smoothness of the Universe. In the case of $\mathbb{R}^{n}$, the only possibility is exotic smooth $\mathbb{R}^{4}$. In this way we found an inherent reason for the 4-dimensionality of the Universe at large scales.

We applied these methods to the spacetime around a Schwarzschild black hole. Due to the unavoidable GR singularity present and QM description required, the smoothness of the solution is modified at large scales. In Kruskal coordinates it results in an exotic black hole as originally discussed in [13]. Finally, we give a concise derivation of the small observed value of the cosmological constant and the expansion rate of the Universe. The Starobinsky Lagrangian is derived from the handle-body structure of exotic $S^{3} \times_{\Sigma} \mathbb{R}, \Sigma=\Sigma(2,5,7)$. The calculations are purely topological, which reflects the power of three- and four-dimensional hyperbolic geometry.

Author Contributions: The authors contributed equally to this work.

Conflicts of Interest: The authors declare no conflict of interest.

\section{References}

1. Van Wesep, R.A. Hidden variables in quantum mechanics: Generic models, set-theoretic forcing, and the appearance of probability. Ann. Phys. 2006, 321, 2453-2475.

2. Boos, W. Mathematical quantum theory I: Random ultrafilters as hidden variables. Synthese 1996, 107, 83-143.

3. Kochen, S.; Specker, E.P. The problem of hidden variables in quantum mechanics. In The Logico-Algebraic Approach to Quantum Mechanics; Springer: Dordrecht, The Netherlands, 1975; pp 293-328.

4. Benioff, P. Models of Zermelo Frankel set theory as carriers for the mathematics of physics. I. J. Math. Phys. 1976, 17, 618-628.

5. Takeuti, G. Two Applications of Logic to Mathematics; Princeton University Press: Princeton, NJ, USA, 1978.

6. Ozawa, M. Scott incomplete Boolean ultrapowers of the real line. J. Symbolic Logic 1995, 60, 160-171.

7. Ozawa, M. Quantum measuring processes of continuous observables. J. Math. Phys. 1984, 25, $79-87$.

8. Klimasara, P.; Król, J. Remarks on mathematical foundations of quantum mechanics. Acta Phys. Pol. B 2015, $46,1309$.

9. Bielas, K.; Klimasara, P.; Król, J. The structure of the real line in quantum mechanics and cosmology. Acta Phys. Pol. B 2015, 46, 2375-2379.

10. Asselmeyer-Maluga, T.; Bielas, K.; Klimasara, P.; Król, J. The latent meaning of forcing in quantum mechanics. Acta Phys. Pol. B 2016, 47, 1685-1690.

11. Król, J. Set theoretical forcing in quantum mechanics and AdS/CFT correspondence. Int. J. Theor. Phys. 2003, 42, 921-935.

12. Król, J. Background independence in quantum gravity and forcing constructions. Found. Phys. 2004, 34, 361-403.

13. Brans, C.H. Localized exotic smoothness. Class. Quant. Grav. 1994, 11, 1785-1792.

14. Birkhoff, G.; von Neumann, J. The Logic of Quantum Mechanics. Ann. Math. 1936, 37, 823-843.

15. Harding, J.; Navara, M. Subalgebras of Orthomodular Lattices. Order 2011, 28, 549-563.

16. Riecan, B.; Neubrunn, T. Integral, Measure, and Ordering; Kluwer Academic Publishers: Dordrecht, The Netherlands, 1997.

17. Bongaarts, P. Quantum Theory: A Mathematical Approach; Springer International Publishing: Houten, The Netherlands, 2015.

18. Lozada, A.; De Sarrazin, G.; Torres, P.L. Complete sets of commuting observables and irreducible sets of observables. Helv. Phys. Acta 1992, 65, 887-897.

19. Blass, A.; Scedrov, A. Freyd's Models for the Independence of the Axiom of Choice; American Mathematical Society: Providence, RI, USA. 1989; p. 134. 
20. Jech, T. Multiple Forcing; Halberstam, H., Wall, C.T.C., Eds.; Cambridge Tracts in Mathematics 88; Cambridge University Press: Cambridge, UK, 1986.

21. Bartoszyński, T.; Judah, H. Set Theory: On the Structure of the Real Line; A.K. Peters: Wellesley, MA, USA, 1995.

22. Li, M.; Li, X.-D.; Wang, S.; Wang, Y. Dark Energy. In Peking University-World Scientific Advance Physics Series; World Scientific: Singapore, Singapore, 2015; Volume 1.

23. Rugh, S.E.; Zinkernagel, H. The Quantum vacuum and the cosmological constant problem. Stud. Hist. Phil. Sci. 2002, 33, 663-705.

24. Ade, P.A.R.; Aghanim, N.; Arnaud, M.; Ashdown, M.; Aumont, J.; Baccigalupi, C.; Banday, A.J.; Barreiro, R.B.; Bartlett, J.G.; Bartolo, N.; et al. Planck 2015 results XIII. Cosmological parameters. Astron. Astrophys. 2016, 594, A13.

25. Ellis, G.F.R.; Maartens, R.; Maccallum, M.A.H. Relativistic Cosmology; Cambridge University Press: Cambridge, UK, 2012.

26. Asselmeyer-Maluga, T.; Brans, C.H. Letter: Cosmological anomalies and exotic smoothness structures. Gen. Rel. Grav. 2002, 34, 1767-1771.

27. Asselmeyer-Maluga, T. Smooth quantum gravity: Exotic smoothness and quantum gravity. In At the Frontier of Spacetime; Asselmeyer-Maluga, T., Ed.; Fundamental Theories of Physics; Springer: Cham, Switzerland, 2016; Volume 183, pp. 247-308.

28. Freedman, M.H. A fake $S^{3} \times \mathbb{R}$. Ann. Math. 1979, 110, 177-201.

29. Asselmeyer-Maluga, T.; Król, J. Inflation and topological phase transition driven by exotic smoothness. Adv. High Energy Phys. 2014, 2014, 867460.

30. Asselmeyer-Maluga, T.; Król, J. On the Origin of Inflation by Using Exotic Smoothness. Arxiv 2016, arXiv:1301.3628.

31. Starobinsky, A.A. A new type of isotropic cosmological models without singularity. Phys. Lett. 1980, 91, 99-102.

(C) 2017 by the authors. Licensee MDPI, Basel, Switzerland. This article is an open access article distributed under the terms and conditions of the Creative Commons Attribution (CC BY) license (http:/ / creativecommons.org/licenses/by/4.0/). 